Classification

Physics Abstracts

02.50.Ga $-02.60 . \mathrm{Cb}-02.70 . \mathrm{Lq}-02.70 . \mathrm{Ns}-47.20 . \mathrm{Hw}$

\title{
Texture Simulation by Lattice Gas from Reaction-Diffusion Models
}

\author{
Luc Decker and Dominique Jeulin \\ Centre de Morphologie Mathématique, École des Mines de Paris, 35 rue Saint-Honoré, \\ 77300 Fontainebleau, France
}

\begin{abstract}
Résumé. - - Les modèles de gaz sur réseau utilisent des particules en mouvement et en interaction sur un graphe. Développés pour simuler des écoulements complexes, ils peuvent être utilisés pour engendrer des structures aléatoires sur des bases physiques. En ajoutant des réactions chimiques entre espèces au déplacement de fluides, des modèles de réaction-diffusion peuvent être engendrés. Cette approche est illustrée par des simulations de textures aléatoires obtenues à partir d'un modèle de création-annihilation et d'un modèle multi-espèces.
\end{abstract}

\begin{abstract}
Lattice gas models use particles moving and interacting on a graph. Developed to simulate complex flows, they can be used to generate random structures on a physical basis. By addition of chemical reactions between species to fluid motion, reaction-diffusion models can be generated. This is illustrated by simulations of random textures obtained from a creation-annihilation model and from a multi-species model.
\end{abstract}

\section{Introduction}

The lattice gas models are a powerful tool for the simulation of complex flows. Starting from simple models with a single species, a hydrodynamic behaviour can be simulated on a grid of points. By addition of marked particles and of aggregation rules, random aggregates (even multiphase) can be generated [1,2]. Other ways of structure generation, introduced in this paper, are obtained by reaction-diffusion models derived from the lattice gas after an introduction of a random reaction operator. This is illustrated by simulations of a creation-annihilation model and of a multi-species model.

\section{Lattice Gas Models}

The lattice gas models enable to implement simulations of flows on a microscopic level. Here particles with a unit velocity and mass move on the vertices of a graph. This model was proposed to solve hydrodynamic problems, mainly in the field of turbulence [3-5]. In this section, the basic rules of construction of these models are given. 
In two dimensions, the FHP-III model proposed by Frisch, Hasslacher and Pomeau (1986) [4] is built on a hexagonal lattice. A population of particles having seven possible velocities (unit velocity with one of the six possible directions on the lattice, or null velocity) moves on the lattice. In what follows, we call a cell each possible particle velocity and each lattice node is thus composed of seven cells. At most, one particle per cell is allowed on every node of the lattice, which corresponds to an exclusion principle. Therefore the gas can be described by a multi-component random set, or by seven binary images (one per velocity). During each time step, every particle moves to its nearest neighbouring node in the direction of its velocity. This results in a translation of the corresponding binary images in the appropriate directions. In addition to the translation of particles, rules of interaction between particles are required, namely collision rules for the particles of a gas. These redistribute the velocities, with the following constraints: preservation of the total mass and momentum at every vertex of the graph. The choice of specific rules enables us to change the viscosity of the fluid for simulations. The process is a sequence of cycles involving the propagation of particles and the redistribution of their velocities. It has been shown [5] that, on a macroscopic scale, the velocity map satisfies the Navier Stokes equations, which allows us to simulate hydrodynamics. The great advantage of this model is its simplicity for implementation, even for complex boundary conditions such as those occurring during the evolution of a microstructure. Since only binary images are used, there is no round-off errors, and the process can be iterated indefinitely. Three dimensional simulations were made possible by the construction of a specific lattice [5].

Boundary conditions imply to know the behaviour of particles when reaching obstacles. If, on a vertex located in the immediate neighbourhood of a solid obstacle, the collision rule is replaced by a bounce-back condition, the simulated fluid respects the usual no-slip condition (the average velocity being equal to zero on the boundary). On the edges of the field, there can be periodic conditions (particles leaving one side of the field are reintroduced with the same velocity on the opposite side) as well as non-periodic conditions, with random injection of particles on the open edges of the field, as already proposed by Jeulin [6].

\section{Building a General Reaction Operator}

Reactions can be introduced in simulations by means of marking particles according to their species and by the addition of a reaction operator. This question was studied in several papers [7-9]. We consider here the most general case of a $k$-species lattice gas model, which is simply obtained by associating one of the $k$ possible species with each particle. Let us call local concentration $n_{\alpha}(t, x)$ of a chemical specie $\alpha$, the exact count of particles of type $\alpha$ at a given lattice node $x$ and at the time $t$ (thus for the FHP-III model, $0 \leq n_{\alpha}(t, x) \leq 7$ ). By extension, the chemical species $\alpha=0$ represents void particles, and $n_{0}$ their concentration.

To the standard lattice gas evolution rule $C \circ T$ (translations $T$ and collisions $C$ ), the following reactive step $R$ is added: at each node $x$, a single cell $i$ is selected uniformly at random. Then, the chemical species $\alpha$ initially found in the cell $i$ may react and change to any other species $\beta$, as a function of the probability $P_{\alpha \beta}\left(n_{\alpha}(t, x)\right)$. This procedure offers the main advantage to avoid computing local concentrations for every possible (or present) species in each node.

In addition, this local reaction operator may be iterated $\nu$ times to balance the diffusion strength in comparison with the reaction rate, so that the lattice gas basic evolution step becomes finally $R^{\nu} \circ C \circ T$. Let us also notice that the operator $R$ alone induces a Markov process depending on the probability matrix $P$. In the present case, a birth and death process (addition or deletion of a single particle at each evolution step) satisfying the exclusion rule of the lattice gas model is used.

In many situations, combinations of such a reactive step with diffusion effects generate complex system behaviours, including spatial self-organization in the form of coexisting areas with distinct concentrations, that are considered as random structures. 


\section{Applications to a Creation-Annihilation Model}

The Schlögl model [10] describes a simple three-molecular auto-catalytic chemical scheme, based on four elementary reactions. A specific cellular automaton model for this particular system has already been proposed by Dab and Boon [7,8], using a two-dimensional square lattice and diffusive collisions produced by particle velocity random rotations. Dab and Boon obtained a simplified microscopic version of the Schlögl model, reduced to a single species micro-dynamic scheme which allows particles to be created or removed, depending on their local concentration. We attempt here to reproduce some of its behaviours from a qualitative viewpoint by means of experimental fittings of the reaction operator parameters.

However, the following general principles will guide this process: at first, a reactive separation towards two distinct stable states is favoured, associated with uniform particle concentrations $\rho_{1}$ and $\rho_{2}$. In addition, the reactive system is initially $(t=0)$ set in an unstable steady state, with concentration $\rho_{0}$ such as $\rho_{1}<\rho_{0}<\rho_{2}$. Higher reaction probabilities are defined for local concentrations ranging from $\rho_{1}$ to $\rho_{0}$ concerning particle annihilation $\left(P_{10}\right)$, and ranging from $\rho_{0}$ to $\rho_{2}$ concerning particle creation $\left(P_{01}\right)$. According to these principles, and since we choose to use the FHP-III lattice gas model, appropriate values are given to the reaction probabilities $P_{01}$ and $P_{10}$, the number $n$ of particles at point $x$ ranging between 0 and 7. In the present case, it is obvious that $n_{0}(t, x)=7-n_{1}(t, x)$. Therefore we both express $P_{01}$ and $P_{10}$ as a function of $n=n_{1}(t, x)$.

By construction, a Markov process (more precisely a birth and death process) is generated on the lattice. For this model, the random number of particles $N$ at point $x$ is characterized by the following transition probability matrix describing the evolution of the population by reaction:

$$
\begin{aligned}
P(n, n-1) & =p_{1} P_{10}(n) \quad \text { with } p_{1}=n / 7, \quad \text { for } n=1,2, \ldots, 7 \\
P(n, n+1) & =p_{0} P_{01}(n) \quad \text { with } p_{0}=1-p_{1}, \text { for } n=0,1, \ldots, 6 \\
P(n, n) & =p_{1} P_{11}(n)+p_{0} P_{00}(n) .
\end{aligned}
$$

The model is completely specified by the 14 free parameters $P_{10}(n)$ and $P_{01}(n)$, which can be reduced to 7 if a symmetric behaviour is simulated $\left(P_{10}(n)=P_{01}(n)\right.$ for $n=1, \ldots, 6$ and $\left.P_{10}(7)=P_{01}(0)\right)$.

One has to point out that, in the framework of the lattice gas models used in the present case, there is a strong coupling between reaction and hydrodynamic processes, since the number of particles per node is proportional to the local pressure. Therefore concentration gradients generate pressure gradients, as would be the case for instance in combustion processes. Results of simulations for a given set of parameters are shown in Figure 1. After a latency stage (until $t \simeq 40$ ), reactions really start from several small areas (seeds) of particle density fluctuations, which derive from local diffusion variations at random lattice nodes. These local de-stabilizations increase, until they generate propagating reactive fronts. At this stage, our system is clearly separated in domains shared out among two different phases $(t=100)$, which form macroscopic random structures - whereas evolution operators are purely local. Finally, one phase prevails over the other one, invading completely the system.

Moreover, the micro-dynamic basis of the lattice gas models allows us to introduce quite easily complex boundary conditions. Indeed, particles are simply bounced back at all particular lattice nodes which are part of obstacle boundaries - in a discrete geometry sense. Thereby, solid obstacles are first added in the system, in the form of boolean random sets [11] with hard-core effects: discs of identical size are sequentially placed in random positions, taking existing disc repulsion areas into account. Such an obstacle model represents classically a given kind of granular media. Effects of this obstacle on the previous reactive system are presented in Figure 2a. It appears in particular that reactive fronts anchor themselves to obstacles, so that they may detect random 


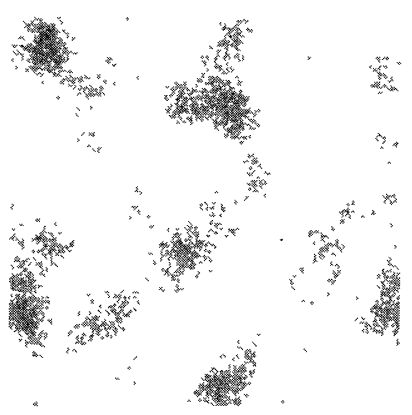

a) $t=0$

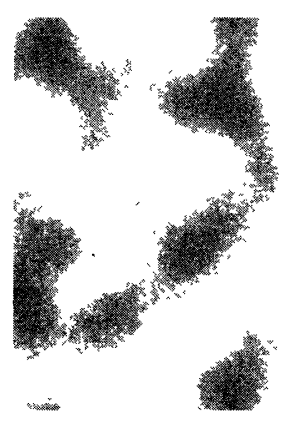

d) $t=80$

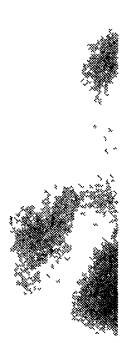

e) $t=100$
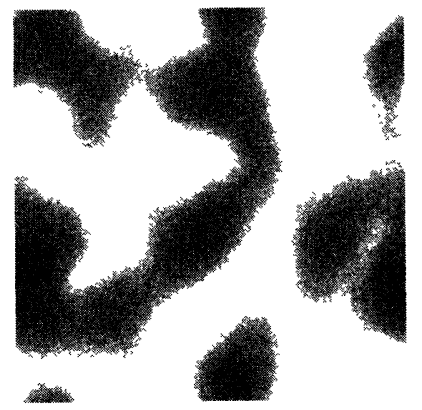

b) $t=40$ c) $t=60$

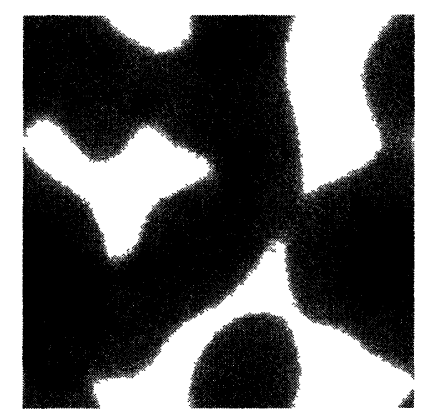

f) $t=150$

Fig. 1. - Pattern formation by a creation-annihilation reactive lattice gas model (based on FHP-III): system evolution during 150 iterations $R^{3} \circ C \circ T$. Grey levels (from light to dark) are proportional to particle count (from 0 to 7) within each node. Lattice size $500 \times 500$ nodes, with periodic boundaries. Initial unstable state density $\rho_{3}=0.20$ particle/cell. Reaction probabilities: $P_{01}(n=3)=0.24, P_{01}(4)=0.08$, $P_{01}(5)=0.04, P_{10}(n=1)=0.15, P_{10}(2)=0.10$.

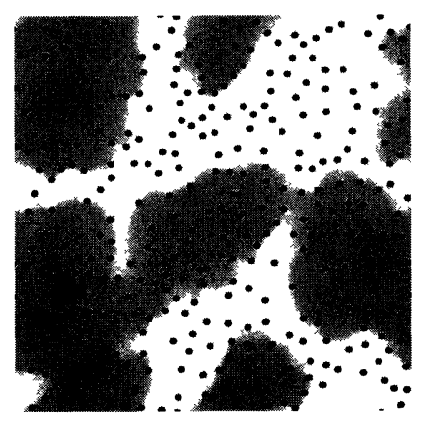

a)

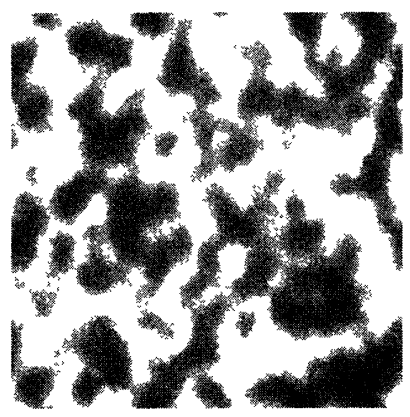

b)

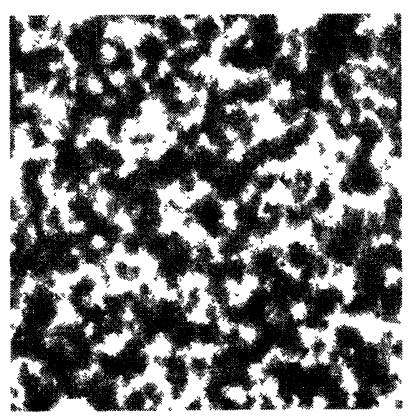

c)

Fig. 2. - Diffusion disturbance effects on reactive front propagation. Same parameters and representation as in Figure 1. In addition, real obstacles are black. a) Granular medium: hard-core model, $t=150 . \mathrm{b}$ ) Scatterers: $15 \%, t=80$. c) Scatterers: $30 \%, t=80$. 
grain alignments. In addition, the front propagation proves to be irregular with time and occurs by successive jumps following immobile steps.

In fact, the presence of obstacles disturbs the standard diffusion process mainly on a macroscopic level. In a second approach, the concept of scatterers is introduced by defining special lattice nodes where particle velocities are reversed at each iteration, exactly in the same way as for obstacle boundaries. But scattering nodes still differ from obstacles because they may hold particles and the reaction operator will apply normally in these nodes. Results of the addition of random scatterers - such as a Poisson point process - on the reactive system studied are shown in Figures $2 b$ and $2 c$, obtained for two different intensities of scattering nodes (expressed as percentages of the whole lattice node count). It appears clearly that scattering nodes bring about a major change of scale of the system, depending on their intensity: the increase of the number of scatterers lowers the effective coefficient of diffusion, resulting into a smaller range of the micro-structure for the same number of time steps (compare Fig. 2b and Fig. 2c).

\section{Applications to a Multi-Species Reactive Decomposition Model}

The creation-annihilation reaction model presented in the previous section produces regions with very different particle densities, which therefore cannot be advected in the same way. As a result, the introduction of particle flows or local particle deflection fields (i.e. conditions with non zero velocity field) may lead to aberrant, non physical results. The example of a basic three-species reactive model presented in this section - in other words, with particles of three distinct colours -, allows only particles to change of colour during the reaction step $R$. In this case, there is a conservation of the total number of particles per node, and therefore there is no transport due to a pressure gradient generated by the reaction. The reaction probabilities are defined so that the species with a low local concentration within a given lattice node may react and change to other possible species, with equal probabilities. Figure 3 presents results for this model. Interesting behaviours are to be noticed, in particular a domain decomposition which is similar to macroscopic surface tension effects. However, the mass is not conserved during the reaction step for a given species. In practice, for this size of simulation field and times of the order of 300000 iterations, a single species usually remains at every lattice node.

Finally, one takes advantage of this new model to observe the consequences of a given particle deflection field on the present reactive decomposition process. Vortex conditions are thus imposed to our system by particle velocity tangential deviations across the surface of a ring centered on the simulation field. With regard to the FHP-III model exclusion principle, all particles on lattice nodes which are uniformly selected may change their velocity so that they fit locally to a vortex velocity vector. The three following cases may occur for each particle: (i) a rotation of its velocity vector through an angle of $\pm \frac{\pi}{3}$, (ii) an immobile particle is set in motion, and (iii) a moving particle is immobilized. The vortex strength thereby depends on the density of nodes subjected to deflection. Such a way of forcing the flow is used by Kadanoff et al. [12]. As shown in Figure 4, the domain morphology is completely changed. Owing to periodic boundary conditions, domains are deformed as a result of the influence of interactions between multiple vortices.

\section{Conclusions}

In this paper, a quite general reaction operator is proposed as an extension to the standard lattice gas model FHP-III. In two applications, our new model exhibits interesting behaviours which are revealed by simulations, such as the formation of macroscopic random structure - including a control of the scale by the coefficient of diffusion - or a domain decomposition by a multi-species reactive model with surface tension effects and deformation capabilities. 


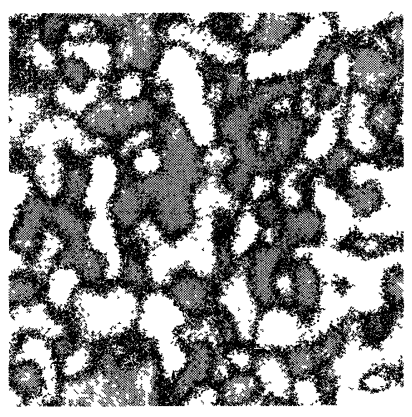

a) $t=400$

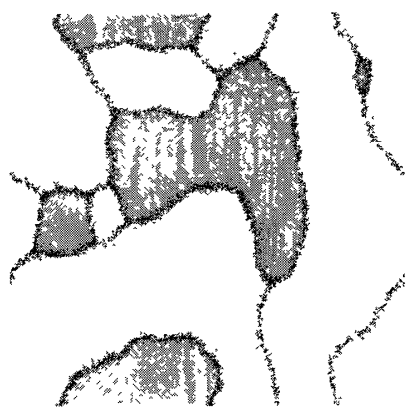

d) $t=7000$

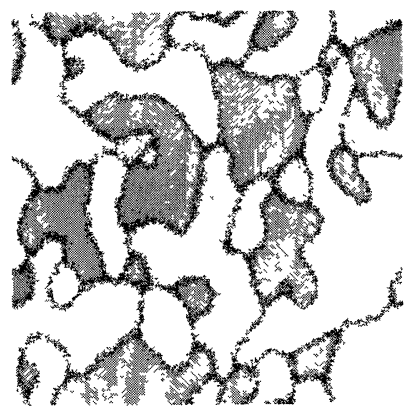

b) $t=1000$

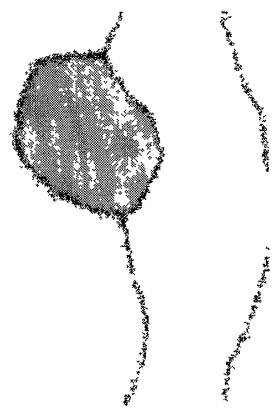

e) $t=26000$

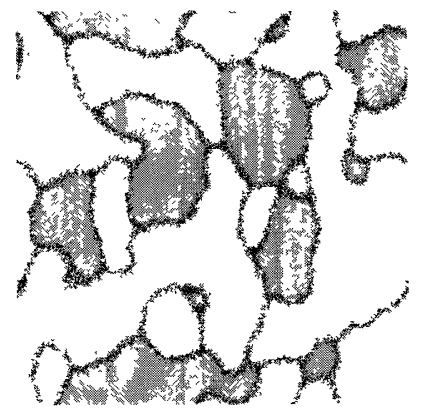

c) $t=2000$

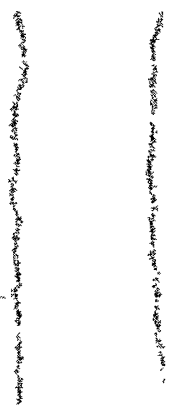

f) $t=80000$

Fig. 3. - Realization of a three-species reactive decomposition lattice gas model $\left(R^{2} \circ C \circ T\right)$. Domains correspond to lattice nodes where more than half of the particles present are of the same species (one distinct grey level for each species), whereas any other species mixtures are indiscriminately represented in black. Lattice size $500 \times 500$ nodes, with periodic boundaries. Initial density: 0.25 particle/cell for each species (numbered 1, 2, 3). Reaction probabilities: $P_{\alpha \beta}\left(n_{\alpha}=1\right)=0.10, P_{\alpha \beta}\left(n_{\alpha}=2\right)=0.05, \forall \alpha \in\{1,2,3\}$, $\beta \in\{1,2,3\} \backslash \alpha$.

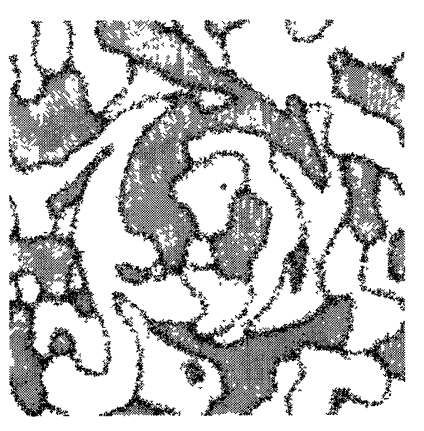

a) $t=1000$

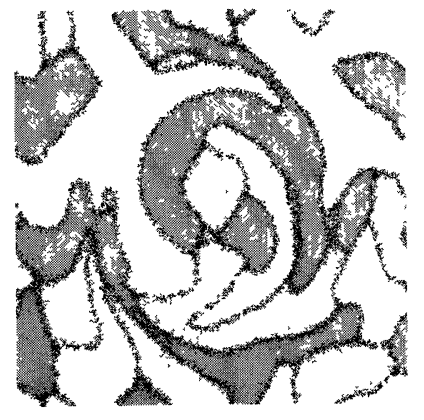

b) $t=2000$

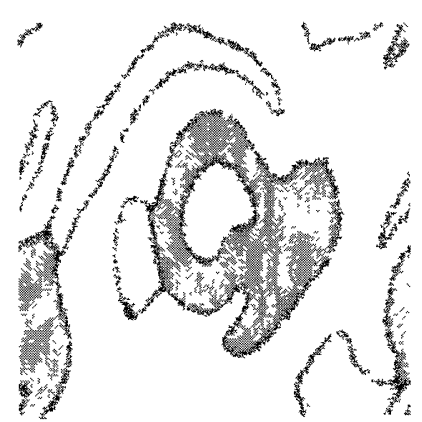

c) $t=7000$

Fig. 4. - Realization of a three-species reactive decomposition lattice gas model with additional vortex conditions. Particle deflection ring inner diameter: 300 nodes, width: 3 nodes. Deflection probability (strength) $p=0.06$. Same parameters and representation as in Figure 3. 
Many types of applications of such models can be explored: catalytic reactions in granular media, effects of a flow on the kinetics of a reactive system, investigation of the behaviour of reaction fronts, as well as germination and growth processes. Competition between reaction and diffusion in real systems can be studied theoretically, and compared to experimental data.

\section{References}

[1] Brémond R. and Jeulin D., Random media and lattice gas simulations, in "Geostatistical Simulations Workshop”, M. Armstrong and P. Dowd, Eds. (Kluwer Academic Publishers, Dordrecht, 1994) pp. 89105.

[2] Brémond R. and Jeulin D., Morphogenesis simulations with lattice gas, in "Mathematical Morphology and its applications to Image Processing", J. Serra and P. Soille, Eds. (Kluwer Academic Publishers, Dordrecht, 1994) pp. 297-304.

[3] Hardy J., de Pazzis O. and Pomeau Y., Phys. Rev. Lett. A 13 (1976) 1949-1961.

[4] Frisch U., Hasslacher B. and Pomeau Y., Phys. Rev. Lett. 56 (1986) 1505-1508.

[5] Frisch U., Lawniczak A., Boon J.P. and Kapral R., Complex Syst. 1 (1987) 649-707.

[6] Jeulin D., Flow and diffusion in random porous media from lattice gas simulations, in "Numerical Methods for the Simulation of Multiphase and Complex Flow", T. Verheggen, Ed. (Springer-Verlag, Berlin, 1992) pp. 106-123.

[7] Dab D. and Boon J.P., Cellular approach to reaction-diffusion systems, in "Cellular Automata and Modelling of Complex Physical Systems", P. Manneville et al., Eds. (Springer-Verlag, Berlin, 1989) pp. 257-273.

[8] Dab D., Lawniczak A., Boon J.P. and Kapral R., Phys. Rev. Lett. 64 (1990) 2462-2465.

[9] Karapiperis T. and Blankleider B., Physica D 78 (1994) 30-64.

[10] Schlögl F., Z. Phys. 253 (1972) 147-161.

[11] Matheron G., Random Sets and Integral Geometry, (Wiley, New York, 1975).

[12] Kadanoff L., Namara G.M. and Zanetti G., Complex Syst. 1 (1987) 791-804. 